\title{
Design of modular fixtures using a 3D-modelling approach
}

\author{
$\underline{\text { U. Farhan }}^{\mathrm{a}}$ and M. Tolouei-Rad \\ ${ }^{a}$ School of Engineering, Edith Cowan University, Western Australia \\ Email: ufarhan@our.ecu.edu.au
}

\begin{abstract}
The need for improved productivity and reduced time to market has been increased significantly in manufacturing processes in recent decades. There are many factors that play a role in providing manufacturing processes with more productivity. One is the use of modular fixtures (MFs). MFs have brought many benefits to manufacturing industries including reduced costs and times of production. An MF can be defined as a complete system that consist different elements for effectively and securely holding the workpiece in place for performing various machining operations. Generally an MF is made by assembling a number of fixture elements in a feasible sequence that is properly determined. Traditionally, the most common method of determination of fixture elements and assembly sequences are making use of 2D drawings and physical models. Obviously these are not efficient methods in the world of advanced manufacturing technologies and there is a vital need for developing more efficient methods. A solution to this is developing a CAD-based 3D-modelling and simulation system to determine the types and of feature elements and the sequence of assembly. Such a system can help fixture designers find the proper MF designs by defining the relationships between fixture elements and workpieces.
\end{abstract}

Designing MFs and fixture elements is a very complex and time-consuming task even by making use of CAD software. A methodology has been developed to simplify this task by determining fixture elements and assembly sequences in a 3D-modelling environment. A fixture elements database is established and the fixture elements are divided into different categories depending on geometries and functions. Currently, MF systems can be constructed from standard MF elements. These elements are pre-manufactured components that include base-plates, clamps, and locators; and they can be re-used after disassembly. These can later be used for other workpieces within a specified range of sizes and shapes.

In order to use these elements effectively, the CAD environment is exploited with developing a database which contains 3D models of standard MF elements. For most workpieces standard MF elements can be extracted directly from the developed database. However, for some workpieces with non-conventional geometries or shapes customised fixture elements should be designed in a 3D CAD environment. A modelling approach is used to find the appropriate MF design that suits the machining processes in need. To do so many parameters are taken into consideration including the number of workpieces to be machined; type of machining operation; locating method; clamping mechanism; and so on.

The methodology developed for MF design has been implemented in a 3D modelling environment by making use of a powerful 3D modelling software, SolidWorks. It makes use of a database which contains 3D models for standard fixture elements. This database is divided into three main categories and each category is divided into different sub-categories depending on the type and function of the MF elements. SolidWorks software provides an excellent 3D environment that can be utilised to model fixture elements and determine assembly processes. This is accomplished by applying mating relationships after considering workpiece setup and fixture elements to achieve the optimum MF assembly design. This paper explains the methodology developed for semi-automated MF design. A case study has been presented to show the validity of the methodology proposed.

Keywords: Modelling, modular fixtures, CAD, design and assembly, database 


\section{INTRODUCTION}

There are many factors playing roles in manufacturing processes in order to improve productivity and reduce production time. Modular fixtures (MFs) are one of the important tools that are widely used to achieve this goal. An MF can be defined as a complete system consisting of different fixture elements used to hold the workpiece in place effectively in order to perform the required machining operations.

In general and on the basis of flexibility, fixture elements (or workholding tools) are classified into general purpose and special purpose workholders (Wang, Rong, \& Li \& Shaun, 2010). Flexibility is of great importance in making use of each type of fixture elements. For instance, the MF shown in Figure 1 provides a flexible workholding method that can be used for holding various workpieces with different shapes (Hoffman, 2004). This design allows the combination of different fixture elements to achieve "possible fixture configurations"(Wang et al, 2010). Because of their flexibility, MFs have become an important factor in flexible manufacturing systems (FMSs). Hoffman classifies MFs into three systems, namely subplate, Tslot, and dowel- pin; where their use depends on the type and geometry of machining operation to be performed (Hoffman, 2004). Standard fixture elements, as shown in Figure 2, are used in MFs. These include baseplates, clamps, risers, and supports (Yi and Bekey, 1996). However, special elements for specific purposes are needed to be designed and the design of these elements is a complex process. The solution for this problem can be found in use of CAD software with $3 \mathrm{D}$ modellingcapabilities.

\subsection{MFs Development}

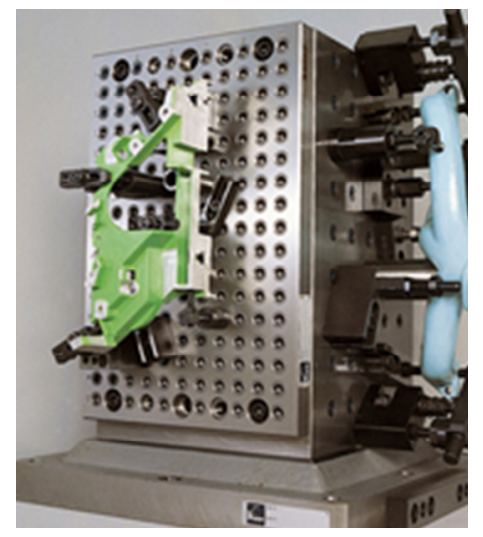

Figure 1. MF model (KIPP catalogues, n.d)

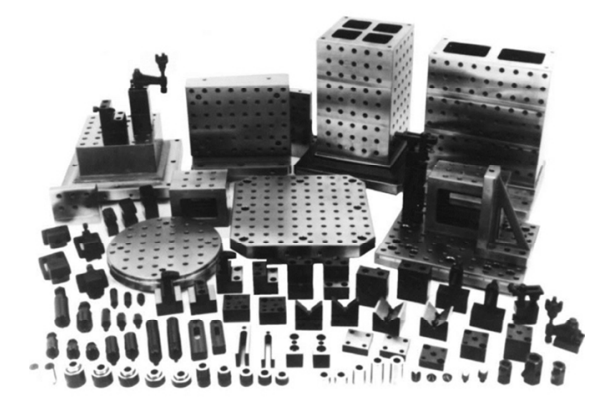

Figure 2. MF elements (Carrlane catalogue, n.d.)

Design of MFs has gained more attention from researchers in the last decades. Some researchers focused on information representation (Mervyn, Kumar \& Nee, 2006 and Gaoliang, Xu, Haiquan, Xin \& Alipour, 2008). Some concentrated on introducing mating relationships to fixture elements in the assembly process. This includes a introducing a method to define the constraints of assembly relationships (Gaoliang et al, 2008). However, due to the need to more automated MF design methods, researchers have utilised CAD software as an important design tool. CAD-based MF designs were developed by using ICAD and UG-2 modelling interfacing (Dai, Nee, Fuh \& Kumar, 1997). This was followed by using 2D AutoCAD drawings (Surendra, Valli, Kumar \& Rao, 2005), and then it was continued by using AutoCAD with 3D modelling (Xiaoling, Yangyi, Jie, Chen, Hua \& Wenlong, 2009).

\subsection{Aims and Significance}

Development and utilisation of a database of fixture elements can be considered as an important factor to make design of MFs more automated. Other factors could be mentioned as the adaption of an appropriate design approach, and utilisation of powerful 3D CAD software to help the designer in performing MF design and assembly process. SolidWorks is CAD software that provides an excellent 3D environment convenient for MF modelling and determining the assembly processes. These three factors are considered as a basis for developing an efficient computer-assisted fixture design (CAFD) system. The aim of this paper is to develop an appropriate methodology by using SolidWorks capabilities for constructing the fixture elements database. 


\section{CAMFD PRINCIPLES}

\subsection{Workpiece Holding Principles}

The specification of the workpiece such as its shape, dimensions and tolerances should be achieved by holing it correctly during machining operations (Chang, Wysk, and Wang, 2006). Other factors also influence the machining outcome including machining operations sequence, cost considerations, direction and strength of cutting forces, and capabilities and orientation of the machine tools (Chang et al., 2006). In terms of holding the workpiece, fixtures are used to locate and clamp it securely in order to perform the required machining operations (Hoffman, 2004).

\section{Locating Principles}

Locating is a crucial factor that should be considered to position and orientate the workpiece in a way that completes the desired machining process thoroughly (Black \& Kohser, 2008). To achieve the desired locating, locators are used to restrict the degrees of freedom (DOF) of the workpiece (Figure 3); these locators should be strong enough against the cutting forces to keep the workpiece in place (Krsulja, Barisic, \& Kudlacek, 2009).

In general, three locating forms can be considered, namely plane, concentric, and radial. For plane locating form, locators are used to locate the workpiece on any surface such as flat, circular or irregular surfaces. For concentric locating form, pin- hole locators are used (Figure 4); and for radial locating form, locators restrict the workpiece movement around the concentric locators (Carrlane catalogue, n.d.). Rong and $\mathrm{Li}$ (1997) divide locating methods into five main categories, namely 3-2-1 principle, plane and pin-hole locating, long-pin locating, V-block, and V-pad locating; yet other classification methods are published in the literature. The 3-2-1 principle is the most commonly method used. In this method, three perpendicular surfaces of the workpiece are used to define the locating position. This involves use of three locating points on the first surface, two points on the second surface, and finally, one locating point on the third surface (Black \& Kohser, 2008)

\section{Clamping Principles}

As the locating is important to restrict the movement of the workpiece, clamping is also crucial to clamp it against the locators and to resist the effects of cutting forces (Cecil, 2001). The direction of clamps should be determined according to cutting forces direction in order to perform machining

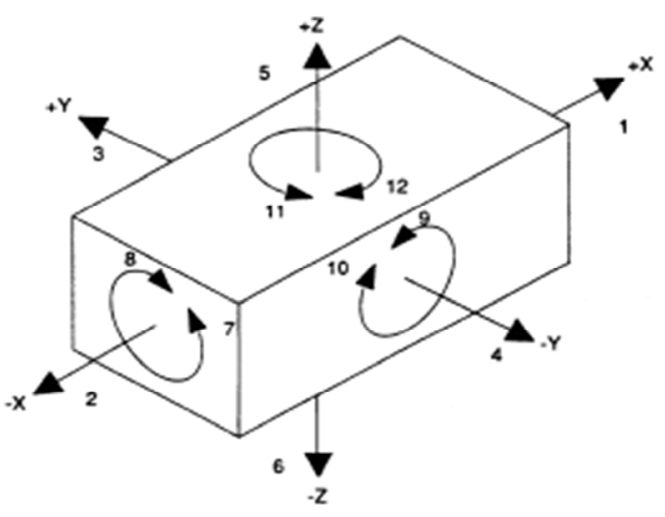

Figure 3. Degrees of freedom (DOF) (Carrlane catalogue, n.d.).

(a) plane location

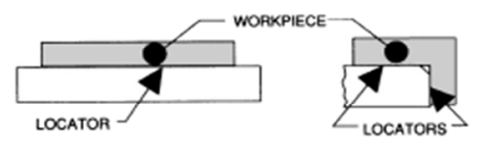

(b) cocentric location

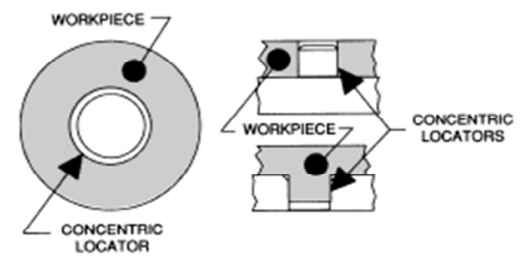

(c) plane location

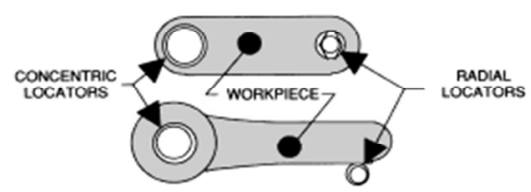

Figure 4. Three locating forms (Carrlane catalogue, n.d.). 
operations securely (Cecil, 2001). As shown in Figure 5, clamping forces are in the same direction of the machining forces which try to push the workpiece down onto thelocators and supporters.(Carrlane catalogue, n.d.).

Moreover, the size of the clamp should be considered in MFs design; the clamp should be large enough to hold the workpiece; yet small enough to stay away of the cutting tool path. There are some other factors that should also be considered in use of clamps. These include machine tool vibration, loads and stresses, damage preventing of the workpiece, and improving loading/unloading speed (Carrlane catalogue, n.d.). For MFs, two major categories are defined for clamping method; these are top and side clamping (Rong and Li, 1997).

\section{Fixture Elements Structure}

The structure of the fixture elements illustrates the relationships between the elements and how they are assembled. In MF design an individual fixture element is to be assembled to other elements, and the relationship between these elements in addition to the purpose of the relationship should be explicitly defined. Moreover, fixture elements are assembled in groups called "functional units"; they consist of one or more of fixture elements (Gaoliang et al, 2008). Functional units can be classified according to their functions into three categories, namely locating, clamping, and supporting units (Gaoliang et al, 2008). Figure 6 shows a typical structure which consists of basic MF components.

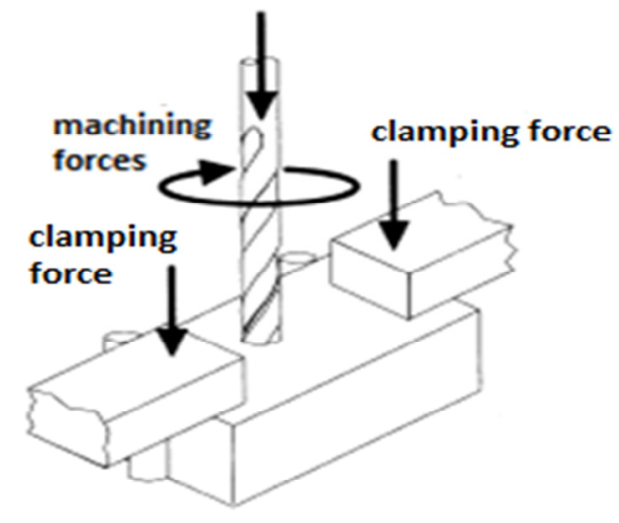

Figure 5. Drilling machining forces (Carrlane catalogue, n.d.)

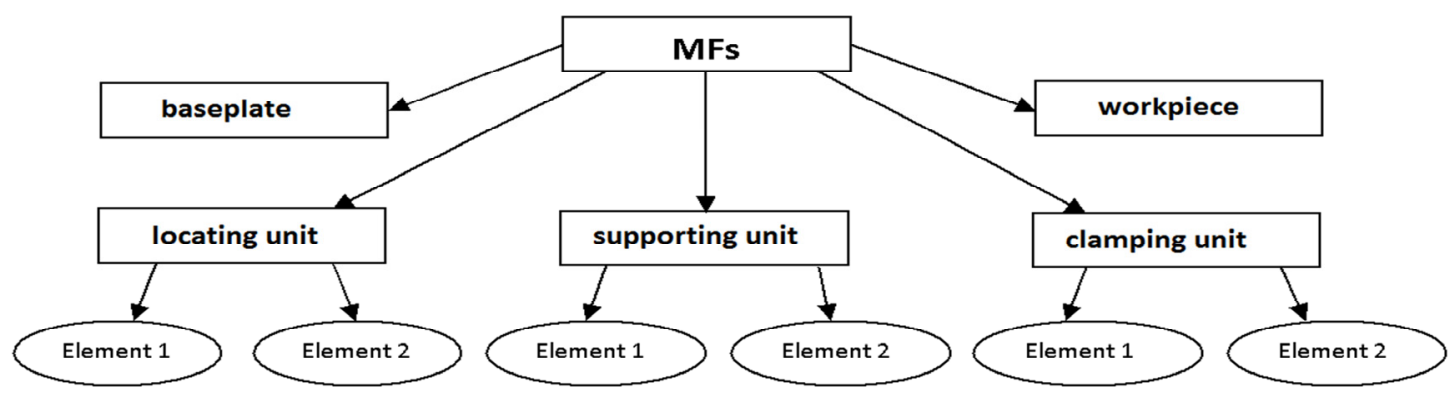

Figure 6. MFs structure (Gaoliang et al, 2008).

\subsection{The Methodology}

In modelling of MFs following three considerations are to be noted:

1. Modelling environment.

2. Workpiece geometry and shape.

3. Machining operations.

In terms of modelling environment, as mentioned before, SolidWorks has been used as the appropriate CAD software to perform MF design process. The workpiece geometry and shape play a role in defining the appropriate MF system, T-slot or dowel-hole, and also defining locating and clamping methods. In this paper, the dowel-hole system has been used and the following steps are taken into consideration: 
Step 1: Analyse workpiece information and machining features, and define workpiece set-up. Locating and clamping surfaces of the workpiece should also be defined.

Step 2: Define the locating method depending on the geometry and the shape of the workpiece.

- Use either 3-2-1 principle or plane-pin hole method for plane surfaces.

- Use long-pin method for a primary inner cylindrical surface.

- Use V-block method for primary external cylindrical surfaces.

- Use V-pad method for secondary external cylindrical surfaces.

Step 3: Select the clamping method by considering the geometry and the shape of the workpiece and the type of machining operations.

Step 4: Select the baseplate from the database depending on the type of the MF system, T-slot or dowel-hole. Then, define the locating and supporting points on the baseplate.

Step 5: Select all fixture elements for the locating and clamping purposes from the database depending on the type of the baseplate determined in Step 4.

Step 6: In SolidWorks modelling environment orientate the workpiece as required considering the baseplate and machining operations, and then assemble the locating and supporting elements on the baseplate.

Step 7: Assemble the workpiece with locating and supporting fixture elements by applying appropriate mating features of SolidWorks.

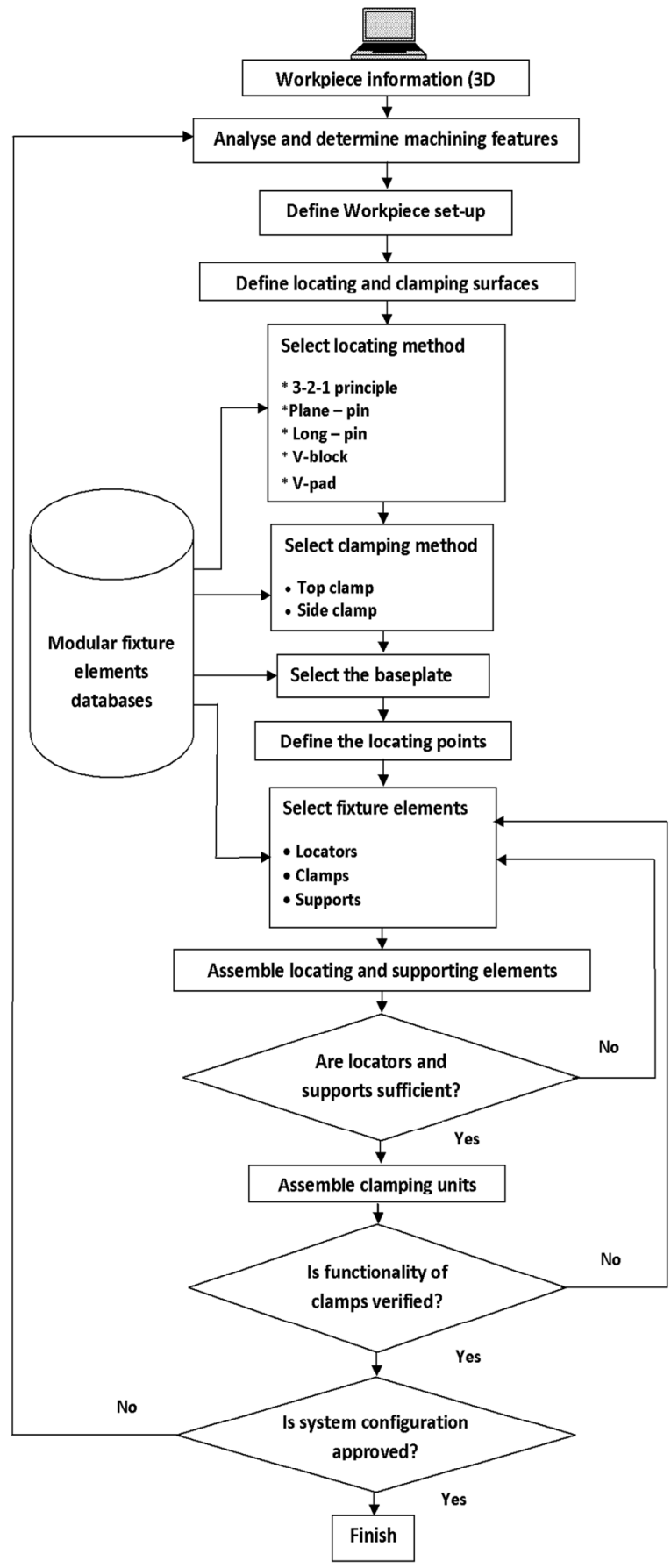

Figure 7. The flow chart of the methodology developed.

Step 8: Construct clamping units and assemble them on the baseplate in order to prevent workpiece movements. It should be noted that there are three types of mate features in SolidWorks, namely standard mates, advanced mates, and mechanical mates. These mate features provide different methods for restricting DOF of the workpiece as required. Figure 7 illustrates the flow chart of the methodology as described above.

\subsection{The Database}

A database has been established in SolidWorks environment and it has been classified into different categories of MF elements such as clamps, locators, and baseplates in order to assist the designer in selecting the elements effectively (Figure 8 (a)). Fixture elements are represented as a list and for each element a 
window is displayed providing details (Figure 8 (b)). The database has been developed in a way that it is easy to reconstruct it to classify fixture elements on the basis of their function and the type, locating and clamping methods, and the class of the standard elements.

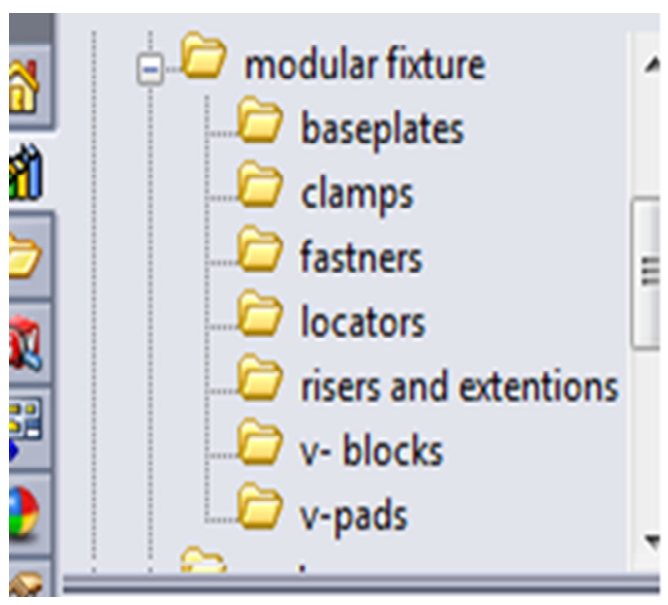

(a)

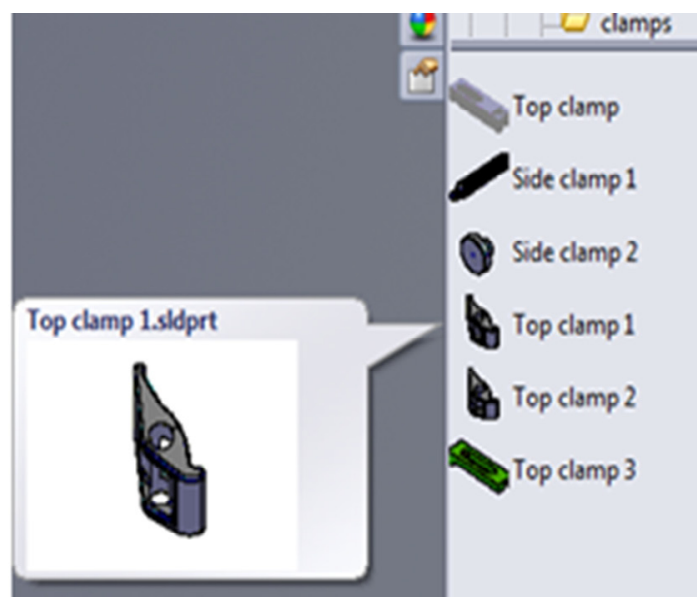

(b)

Figure 8. (a) Different categories of the database, (b) details of the fixture elements.

\section{CASE STUDY}

CAMFD has been used to design MFs for two different parts, one prismatic and one rotational. V-blocks are used as locators for the rotational part, while the 3-2-1 principle is used for the prismatic part. Both examples verify the validity of the methodology developed. The MF for prismatic part has been designed to be used with quick change subplates (Figure 9 (a)); while for the rotational part the MF has been designed to make it possible to hold more than one part at the same time (Figure 9 (b)).

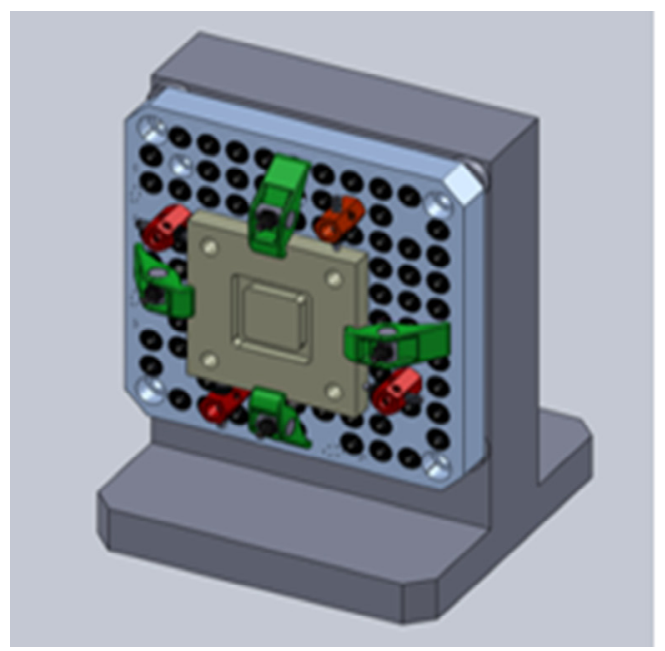

(a)

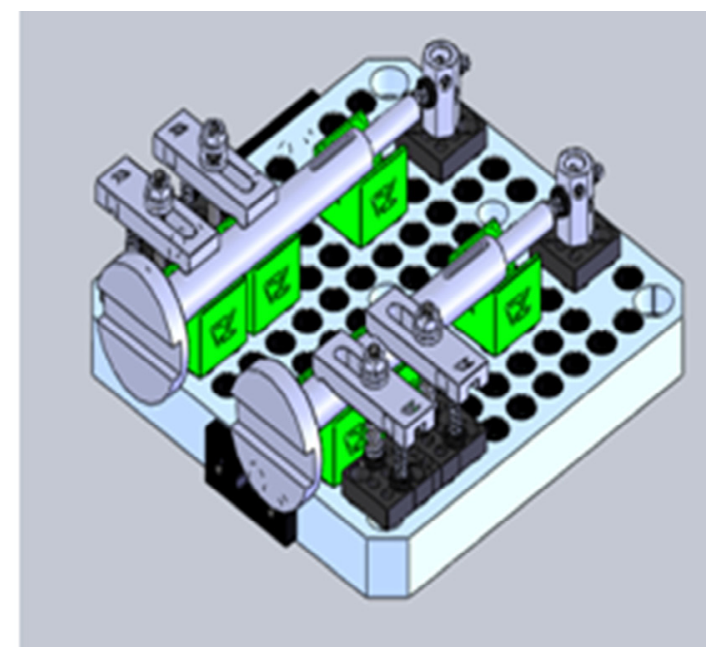

(b)

Figure 9. (a) Quick change subplate system, (b) MF for rotational parts.

\section{DISCUSION AND CONCLUSIONS}

The flexibility of MFs plays an important role in reducing machining costs and the times in CNC machines. The CAD-based methodology for MF design explained in this paper can help improve productivity of this task significantly, lowering the time and skill level needed. The system developed makes use of a powerful 
3D modelling software making it possible to select appropriate fixture elements including clamps, supports, and locators, and define relationships between these elements. This makes it possible to detect any inconsistencies, interferences of fixture elements, and possible collisions of the moving cutting tools with fixture elements before the actual fixture is made. The database of fixture elements developed in this work plays a central role in the design process and saves a lot of time at design stage.

The two examples presented employ top-clamping method; however, different types of clamps are used. Forged clamps are selected for the quick change subplate while strap clamps are used for the rotational part. In both examples side supporters are used to restrict workpiece movements.

\section{REFERENCES}

Black, J. T., and Kohser, R. A. (2008). Materials and processes in manufacturing. The USA: John Wiley and Sons.

Carrlane catalogue. [n.d.]. Available from http://www.carrlane.com/catalog/index.cfm

Cecil, J. (2001). A clamping design approach for automated fixture design. Advanced Manufacturing Technology, 18, 784-789.

Chang, T. C., Wysk, R. A., and Wang, H. P. (2006). Computer-aided manufacturing. New Jersey: Pearson Education, Inc.

Dai, J. R., Nee, A. Y., Fuh, J. Y. H., and Kumar, A. S. (1997). An approach to automating modular fixture design and assembly. ProQust Science Journals, 509-521.

Gaoliang, P., Xu, H., Haiquan, Y., Xin, H., and Alipour, K. (2008). Precise manipulation approach to facilitate interactive modular fixture assembly design in a virtual environment. Assembly Automation, $28,3,216-224$.

Hoffman, E. G. (2004). Jig and fixture design ( $5^{\text {th }}$ ed.), New York: Delmar Learning.

Krsulja, M., Barisic, B., and Kudlacek, J. (2009). Assembly setup for modular fixture machining process. Advanced Engineering, 3,1, 39-51.

Rong, Y., and Li, X. (1997). Locating method analysis based rapid fixture configuration design. IEEE, 27-32.

Mervyn, F., Kumar, A. S., and Nee, A. Y. C. (2006). Fixture design information support for integrated design and manufacturing. International Journal of Production Research, 44, 11, 2205-2219.

Surendra, B. B., Valli, P. M., Kumar, A. V. V. A., and Rao, D. N. (2005). Automatic modular fixture generation in computer- aided process planning systems. ProQuest Science Journals, 1147-1152.

The KIPP catalogues. [n.d.]. Available from http://www.kipp.com/cms/wm?catld=1620\&artld=4171

Wang, H., Rong, Y., Li, H., and Shaun, P. (2010). Computer-aided fixture design : Recent research and trends. Computer-Aided Design, 42, 1085-1094.

Xiaoling, K., Yangyi, Jie, Z., Chen, G., Hua, Z., and Wenlong, Z. (2009). Research and development of the software on computer-aided fixture designing. IEEE, 9, 1233-1236.

Yi, C., and Bekery, A. (1996). Assembly planning for modular fixtures. IEEE, 96, 704-711. 